\title{
Gezielte Injektion von Effektoren durch Kontrolle der Proteindynamik
}

STEPHAN WIMMI, FLORIAN LINDNER, ANDREAS DIEPOLD

ABTEILUNG ÖKOPHYSIOLOGIE, MAX-PLANCK-INSTITUT FÜR TERRESTRISCHE

MIKROBIOLOGIE, MARBURG

The type III secretion system (T3SS) enables direct injection of bacterial effector proteins into eukaryotic cells. We found that the dynamic cytosolic interface of the system allows Yersinia enterocolitica to suppress premature secretion at low $\mathrm{pH}$, ensuring rapid activation at the site of action. Exploiting this principle, we developed a light-controlled T3SS based on optogenetic interaction switches, which provides unprecedented spatiotemporal control of protein secretion and translocation.

DOI: $10.1007 / \mathrm{s} 12268-021-1667-4$

(C) Die Autoren 2021

Bakterien setzen eine Vielzahl molekularer Werkzeuge ein, um Wirtsorganismen zu infizieren, Symbiosen einzugehen oder in der Auseinandersetzung mit anderen Bakterienspezies die Oberhand zu gewinnen. Vom filigranen Injektionsapparat bis zum massiven Rammbock gehören diese Werkzeuge zu den komplexesten und spannendsten Strukturen, die Bakterien produzieren.

Das Typ-III-Sekretionssystem (T3SS), auch bekannt als Injektisom, erlaubt den direkten Transfer von Effektorproteinen aus dem bakteriellen Cytosol in eukaryotische Zellen (Abb. 1). Sein Einsatz ist unverzichtbar für bedeutende Krankheitserreger wie

- Abb. 1: Das Typ-III-Sekretionssystem (T3SS). Einsatz oben, Visualisierung der Proteindynamik durch single particle tracking von PAmCherry-SctQ in lebenden Yersinia enterocolitica. Einzelne Proteine werden gemäß ihrer Bewegung als mobil (blau) oder gebunden (rot) eingestuft und unterscheiden sich klar in ihrer Position im Bakterium. Hauptbild, Prinzip der Lichtsteuerung der T3SS-Aktivität durch Integration eines optogenetischen Schalters. Im gezeigten Beispiel (LITESEC-act) wird das im Dunkeln (rechts) an die Membran gebundene T3SS-Protein SctQ durch blaues Licht freigesetzt (links). Dadurch kann es mit dem T3SS interagieren und die Proteinsekretion aktivieren. Teilweise modifiziert aus [1, 5].
Salmonellen, Shigellen oder auch das Pestbakterium Yersinia pestis. Gereinigte Subkomplexe und in situ-Strukturanalyse erlauben faszinierende Einblicke in den Aufbau des Injektisoms.
Dennoch ist noch immer weitgehend unbekannt, wie Selektion und Export der Effektoren in den Bakterien bewerkstelligt und gesteuert werden. In den letzten Jahren konnten wir hier entscheidende Fortschritte erzielen und zeigen, dass die cytosolischen Komponenten des Injektisoms eine dynamische Schnittstelle darstellen. Bakterien nutzen diese Dynamik für eine schnelle Regulation der Funktion des T3SS. Der Einsatz optogenetischer Schalter ermöglicht es sogar, die Dynamik für die lichtabhängige Kontrolle des Effektorexports durch das Injektisom einzusetzen.

\section{Yersinien als Modell für das T3SS}

Unser Labor arbeitet mit einem Verwandten des Pesterregers, dem Darmpathogen Yersinia enterocolitica. Yersinien sind ein beliebtes Modellsystem für das T3SS, da sich die Sekretion hier nicht nur durch Kontakt zu Wirtszellen, sondern auch einfach über die

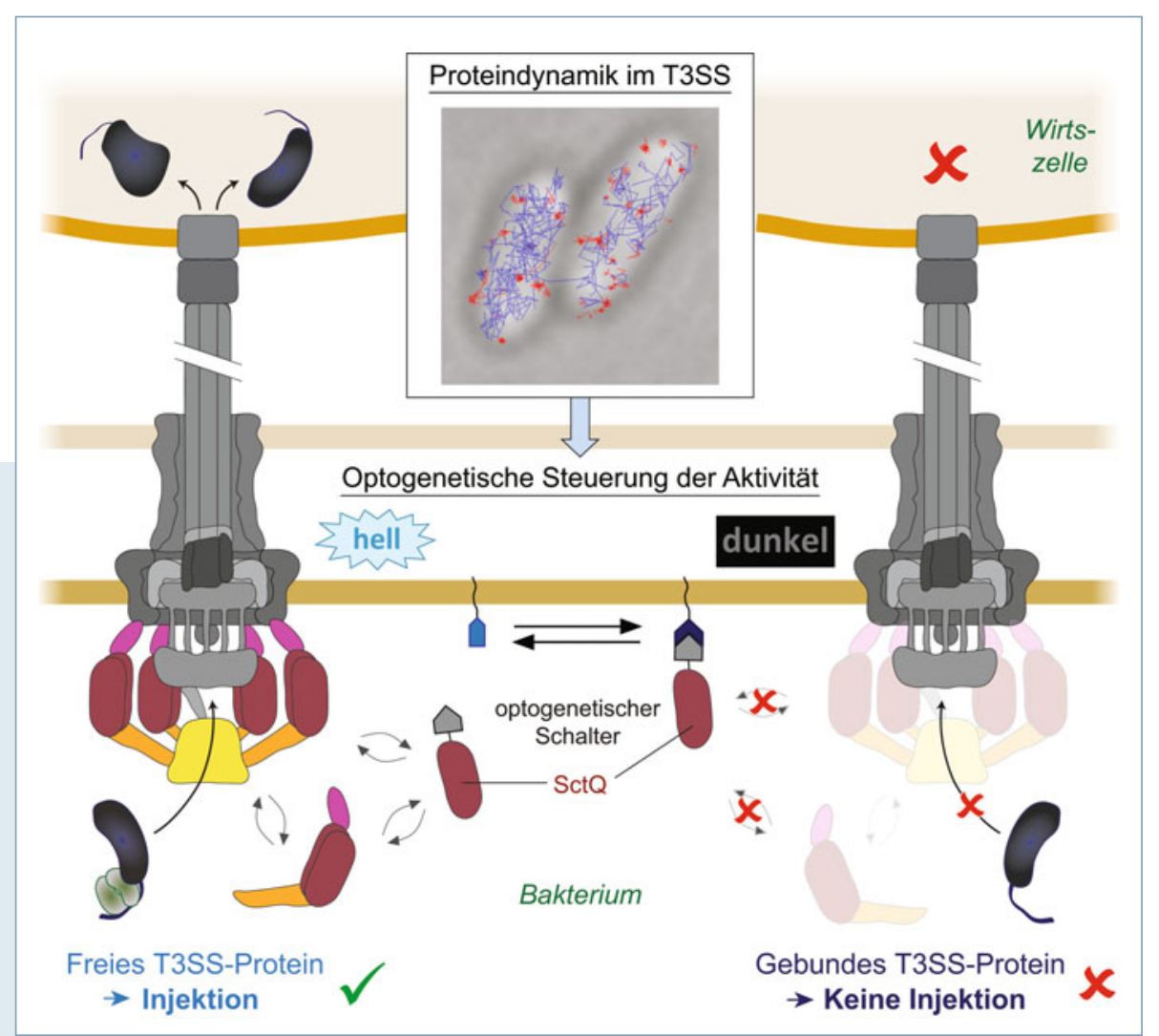




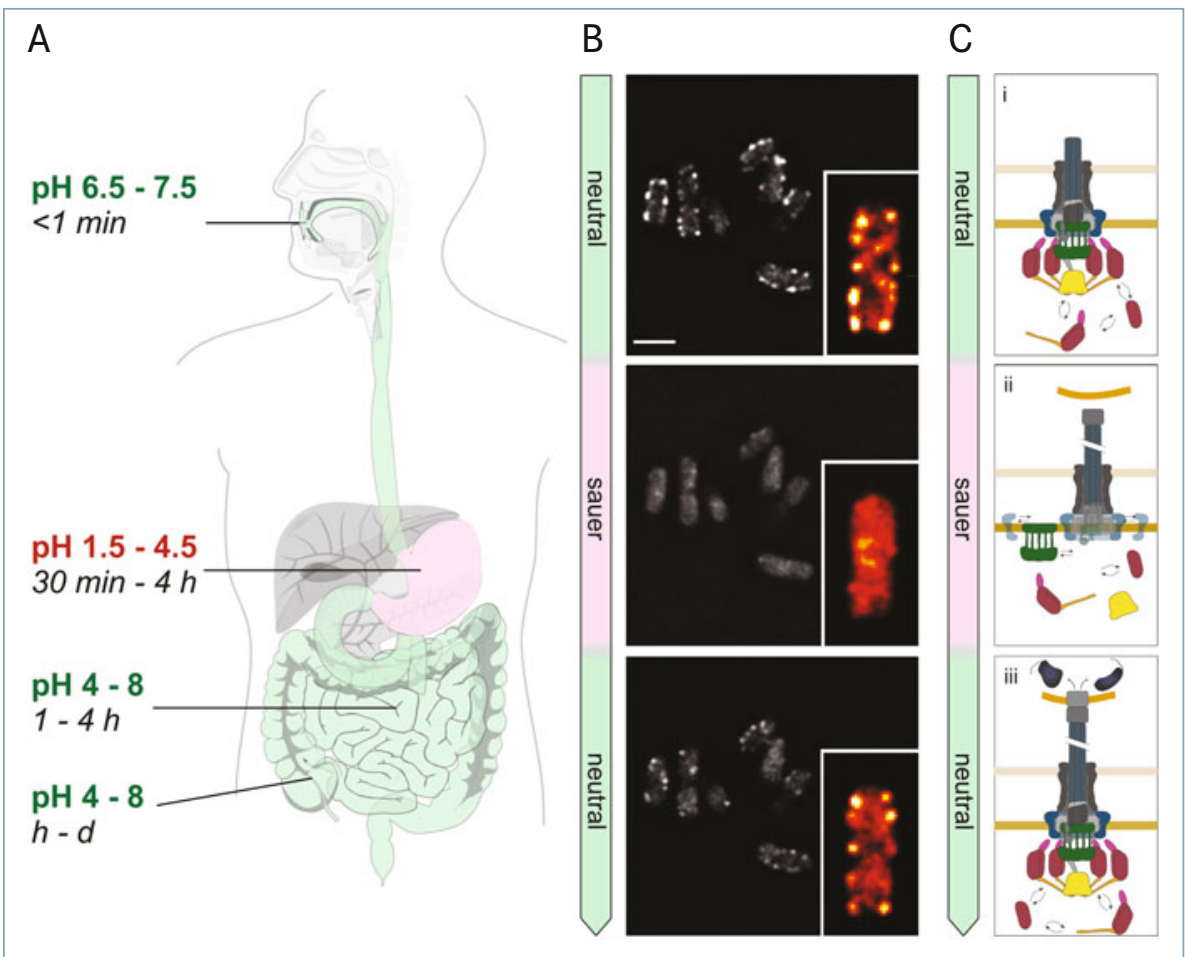

$\Delta$ Abb. 2: Die Dynamik des T3SS erlaubt seine Anpassung an wechselnde äußere Bedingungen. A, pH-Wert entlang des Infektionswegs von Yersinia enterocolitica im menschlichen Verdauungstrakt. B, temporäre Relokalisierung der T3SS-Komponente EGFP-SctK vom Injektisom (Fluoreszenzfoci bei pH 7) in das Cytosol in saurer Umgebung ( $\mathrm{pH} 4$ ). Maßstab: $2 \mu \mathrm{m}$. C, schematische Darstellung der pH-Anpassung des T3SS, beteiligte Proteine in Farbe: i: Aufbau des T3SS nach Eintritt in den Wirtskörper; ii: teilweise Dissoziation der Membrankomponente SctD (blau) und resultierende Ablösung der cytosolischen Komponenten bei $\mathrm{pH} 4$ verhindert die Proteinsekretion; iii: Bindung von SctD und der cytosolischen Komponenten bei neutralem pH-Wert ermöglicht die rasche Aktivierung des T3SS. Modifiziert aus [2].

Calciumkonzentration des Mediums steuern lässt. Um die Funktion und Steuerung des T3SS in lebenden Bakterien und im Kontakt zu Wirtszellen untersuchen zu können, nutzt unser Labor eine große Bibliothek fluoreszenzmarkierter T3SS-Komponenten. Diese ermöglichen Einblicke in den Aufbau, die zelluläre Verteilung, aber auch die Dynamik des gesamten Systems.

\section{Der dynamische Injektionsapparat}

Der in der Membran verankerte Basalkörper des T3SS - aus Membranringen samt Nadel - ist stabil und kann als Ganzes aufgereinigt werden. Im Gegensatz dazu sind die Bestandteile des cytosolischen Komplexes auf der Innenseite des Injektisoms nicht statisch an den Basalkörper gebunden, sondern stehen im permanenten Austausch mit einem cytosolischen Pool (Abb. 1B). Dabei erfolgt dieser Austausch nicht ungeregelt, sondern steht in direkter Verbindung zur Aktivität des T3SS [1]. Aber was haben die Bakterien von dieser Dynamik?

\section{Darmpathogene unterdrücken} Proteinsekretion in saurer Umgebung

Das T3SS ist ein adaptives System, das Veränderungen in der Umgebung wahrnimmt und darauf reagiert. Ein Temperaturanstieg auf $37{ }^{\circ} \mathrm{C}$, die Körpertemperatur der Wirtsorganismen, leitet Expression und Aufbau des Injektisoms ein. Auch der Sauerstoffgehalt und, beim von intrazellulären Salmonellen eingesetzten SPI-2-T3SS, der pH-Wert des umgebenden Lysosoms werden als Indikatoren für die Aktivierung des T3SS genutzt. Auch Y. enterocolitica als Darmpathogen ist auf dem Weg durch den menschlichen Körper einem Wechselbad verschiedener $\mathrm{pH}$ Werte ausgesetzt (Abb. 2A). Nach Passage der M-Zellen vom Dünndarm ins Lymphsystem kommt es zum Kontakt mit Makrophagen - hier muss die bakterielle Waffe des T3SS einsatzbereit sein.

Wir fragten uns, ob Y. enterocolitica genau diesen $\mathrm{pH}$-Unterschied nutzt, um die Effektorsekretion, die bei Zellkontakt ansonsten recht unspezifisch aktiviert wird, zu regulie- ren. Tatsächlich zeigte sich, dass in saurer Umgebung zwar die Nadeln außerhalb der Zelle stabil sind, aber keine Effektoren exportiert werden. Dieser Stopp des Proteinexports geht einher mit einem Verlust jeglicher Bindung der Komponenten des cytosolischen Komplexes an die verbleibende Injektisomstruktur. Sobald der pH-Wert der Umgebung wieder neutral ist, binden genau diese Proteine unmittelbar wieder an ihrer ursprünglichen Position am Injektisom (Abb. 2B). Auf der Suche nach der molekularen Grundlage dieses Mechanismus konnten wir zeigen, dass der Umgebungs-pHWert direkt erkannt wird: SctD, ein Membranprotein, das normalerweise einen zentralen Ring des Injektisoms bildet, dissoziiert in saurer Umgebung teilweise und verhindert so die Bindung der cytosolischen Komponenten.

Was ist der physiologische Mehrwert dieses Mechanismus? Das Verhindern der Sekretion am falschen Ort spart Energie und minimiert die Risiken einer Erkennung durch das Immunsystem; vor allem aber ist das Injektisom durch die temporäre Inaktivierung viel schneller bereit, in $\mathrm{pH}$-neutraler Umgebung auf Wirtszellen zu reagieren (und diese durch gezielte Effektorinjektion zu deaktivieren), als dies bei einer Neusynthese an Ort und Stelle der Fall wäre.

Da die schnelle und präzise Aktivierung der Sekretion für alle Bakterien, die das Injektisom benutzen, von größter Bedeutung ist, lag die Frage nahe, ob der Einfluss des externen pH-Werts eine Spezialisierung von Y. enterocolitica oder ein allgemeiner Mechanismus in Gastrointestinalerregern ist. Hierfür verglichen wir die Lokalisation der cytosolischen Komponenten im Darmpathogen Shigella flexneri sowie in Pseudomonas aeruginosa, das als Wunderreger den Magen bei einer Infektion meist nicht passiert. Trotz der größeren evolutionären Distanz zeigt S. flexneri eine ähnliche $\mathrm{pH}$-abhängige Lokalisierung der cytosolischen Komponenten wie Y. enterocolitica, im Gegensatz zu P. aeruginosa. Unsere Ergebnisse legen nahe, dass Darmerreger die reversible pH-abhängige Deaktivierung des Injektisoms gezielt nutzen, um eine spezifische und schnelle Aktivierung der Effektorsekretion am richtigen Ort zu ermöglichen (Abb. 2C, [2]).

\section{Präzise optogenetische Steuerung der Proteininjektion}

Das T3SS, von der Evolution auf die effektive Translokation von Effektoren in Wirtszellen 
Abb. 3: Lichtgesteuerte Apoptose von HeLa-Zellen mittels T3SS-abhängiger Translokation des proapoptotischen Proteins tBID. Apoptotische Zellen sind als sternförmig kondensierte Zellen zu erkennen; ihr Anteil ist unten quantifiziert. Maßstab: $50 \mu \mathrm{m}$. Es wurden jeweils mehr als 1.000 Zellen aus fünf unabhängigen Experimenten untersucht; als Kontrollen wurden ein ungesteuerter Wildtyp-Stamm (WT) und eine T3SS-inaktivierte Mutante $(\Delta \mathrm{Sct} Q)$ verwendet. Einzelpunkte entsprechen dem Prozentsatz der apoptotischen Zellen in einzelnen Sichtfeldern; Fehlerbalken zeigen den Standardfehler des Mittelwerts an. $* / * * *: p<0,05 / 0,001$ in einem zweiseitigen homoskedastischen t-Test; n. s.: Unterschied nicht statistisch signifikant $(p=0,40)$. Modifiziert aus [5].

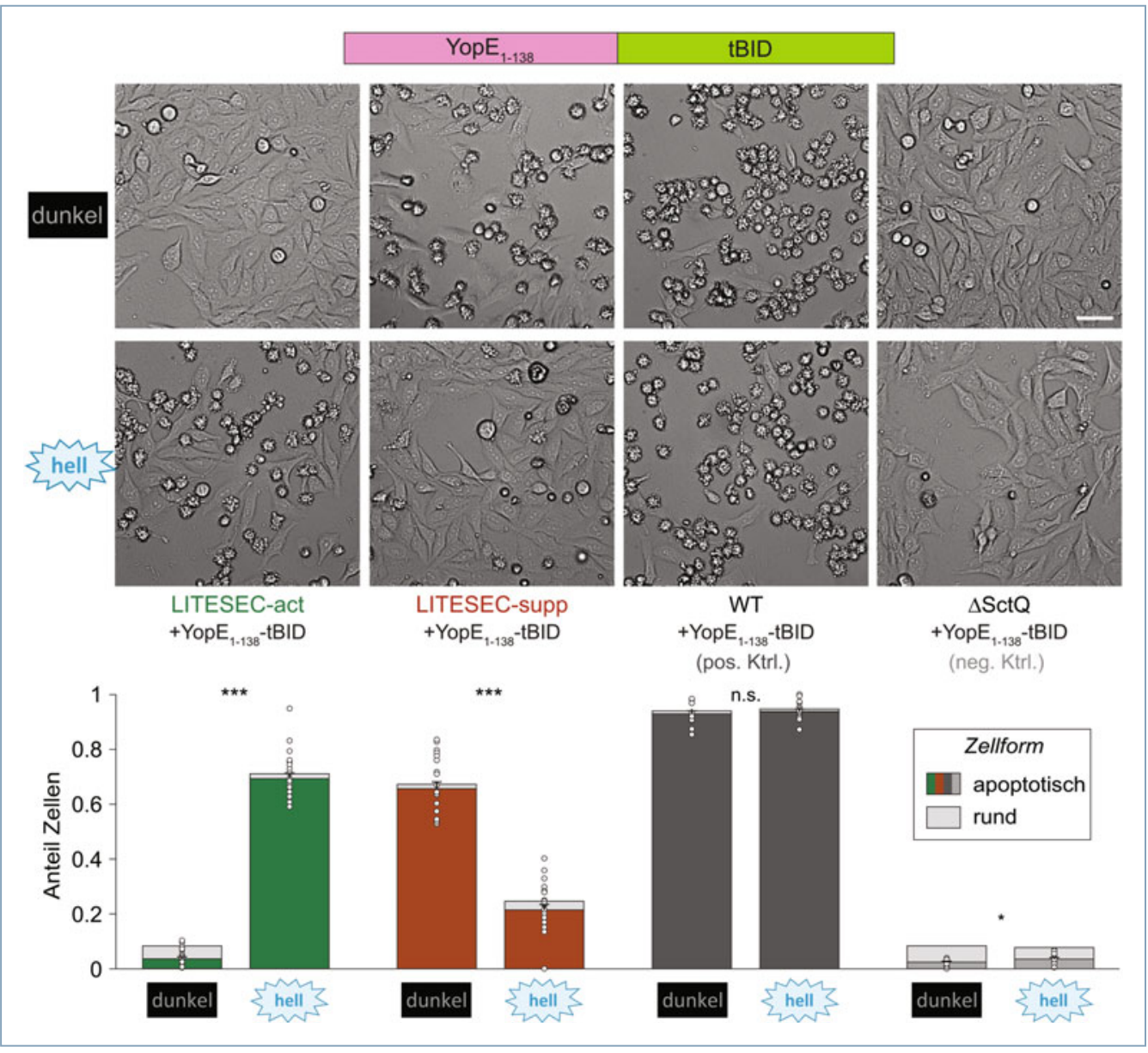

getrimmt, ist ein elegantes Werkzeug, um gezielt Proteine in eukaryotische Zellen zu bringen. Allerdings wird das System durch Wirtszellkontakt relativ unspezifisch aktiviert. Für therapeutische Anwendungen, vor allem für die Injektion potenter proteinbasierter Wirkstoffe, ist dies ein großes Problem, da durch die Mobilität der Trägerbakterien unerwünschte Wirkungen an anderen Orten im Körper auftreten könnten. Eine präzise räumliche und zeitliche Kontrolle der Effektorsekretion ist daher eine Grundvoraussetzung, um aus dieser generellen bakteriellen Waffe ein hochspezifisches Werkzeug für den Einsatz in Medizin und Biotechnologie zu machen.

Die Erkenntnis, dass die Dynamik der cytosolischen Komponenten von Bakterien dazu genutzt wird, die Aktivität des T3SS zu kontrollieren, legte nahe, dass mit diesem Prinzip auch eine gezielte Steuerung möglich sein könnte. Zu diesem Ziel integrierten wir optogenetische Schalter - Proteinpaare, deren Interaktion durch Licht induziert oder aufgehoben werden kann - in das T3SS. Mithilfe dieser Schalter wollten wir eine essenzielle dynamische cytosolische Komponente des Injektisoms, SctQ, mit einem AnkerKöder-System gezielt an der Membran binden und wieder freisetzen (Abb. 1). Der Effekt wäre folglich eine lichtgesteuerte Aktivierung bzw. Deaktivierung der Proteinsekretion und -translokation.

Als Grundsystem wählten wir die optogenetischen Schalter LOVTRAP und iLID, deren Komponenten bei Beleuchtung dissoziieren bzw. assoziieren [3, 4]. In beiden Fällen verankerten wir einen der Interaktionspartner in der Membran von $Y$. enterocolitica („Anker“) und fusionierten den anderen mit SctQ („Köder“). Tatsächlich zeigen unsere Versuche, dass das resultierende „LITESECT3SS“-System (für light-induced translocation of effectors through sequestration of endogenous components of the T3SS) es erlaubt, die Aktivität des T3SS gezielt durch blaues Licht zu aktivieren oder zu unterdrücken (Abb. 3 [5]).

Da das T3SS, und damit LITESEC, nicht auf seine natürlichen Effektoren beschränkt ist, lassen sich damit auch therapeutische Proteine sekretieren. So konnten wir das proapoptotische Protein tBID an ein spezifisches Sekretionssignal koppeln und als künstli- chen Effektor binnen weniger Minuten lichtgesteuert in eukaryotische Zellen injizieren, was in kurzer Zeit zum kontrollierten Zelltod führte (Abb. 3C).

\section{Dynamik und optogenetische Steuerung biologischer Prozesse}

Diese Ergebnisse zeigen eindrucksvoll die Rolle des Proteinaustauschs in der Regulation des T3SS. Dynamische Prozesse dieser Art finden sich in allen Bereichen der Biologie [6], ihre Bedeutung ist jedoch häufig noch unerforscht. Während unserer Arbeiten zum LITESEC-System wurde uns klar, dass die optogenetische Steuerung über praktische Anwendungsbeispiele hinaus auch vollkommen neue Aspekte der Biologie zugänglich macht. Die präzise und minimalinvasive Kontrolle der Verfügbarkeit einzelner Proteine erlaubt es, die Funktion auch essenzieller Prozesse in vivo und in Echtzeit zu beobachten und zu beeinflussen. Insbesondere für anpassungsfähige und vernetzte Systeme wie bakterielle Virulenzfaktoren - ist die Optogenetik damit ein effektives Tool für die praktische Anwendung wie auch die Grundlagenforschung. 


\section{Danksagung}

Wir danken der Max-Planck-Gesellschaft sowie der Deutschen Forschungsgemeinschaft (DI 1765/5) für finanzielle Unterstützung und allen Mitgliedern des Labors für hilfreiche Diskussionen und gute Zusammenarbeit.

\section{Literatur}

[1] Diepold A, Kudryashev M, Delalez NJ et al. (2015) Composition, formation, and regulation of the cytosolic C-ring, a dynamic component of the type III secretion injectisome. PLOS Biol 13: e1002039

[2] Wimmi S, Balinovic A, Jeckel H et al. (2021) Dynamic relocalization of cytosolic type III secretion system components prevents premature protein secretion at low external pH. Nat Commun 12: 1625
[3] Wang H, Vilela M, Winkler A et al. (2016) LOVTRAP: an optogenetic system for photoinduced protein dissociation. Nat Methods 13: 755-758

[4] Guntas G, Hallett RA, Zimmerman SP et al. (2015) Engineering an improved light-induced dimer (iLID) for controlling the localization and activity of signaling proteins. Proc Natl Acad Sci $112: 112-117$

[5] Lindner F, Milne-Davies B, Langenfeld K et al. (2020) LITESEC-T3SS - light-controlled protein delivery into eukaryotic cells with high spatial and temporal resolution. Nat Commun 11: 2381

[6] Tusk SE, Delalez NJ, Berry RM (2018) Subunit exchange in protein complexes. J Mol Biol 430: 4557-4579

Funding note: Open Access funding enabled and organized by Projekt DEAL. Open Access: Dieser Artikel wird unter der Creative Commons Namensnenn 4.0 International Lizenz veröffentlicht, welche die Nutzung, Vervielfältigung, Bearbeitung, Verbreitung und Wiedergabe in jeglichem Medium und For (a)

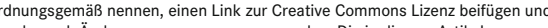
enthaltenen Bilder und sonstiges Drittm wren. De in diesem Artkel genannten Creative Commons Lizenz, sofern sich aus der Abbildungslegende nichts anderes ergibt. Sofern das betreffende Material nicht unter der nz steht und die betreffende Handlung nich Weiterverwendungen des Materials die Einwilligung oben aufgeführt

Rechteinhabers einzuholen. Weitere Details zur Lizenz entnehmen Sie bitte der Lizenzinformation auf http://creativecommons.org/licenses/by/4.0/deed.de.

Dr. Andreas Diepold

Abteilung Ökophysiologie

Max-Planck-Institut für terrestrische

Mikrobiologie

Karl-von-Frisch-Straße 10

D-35043 Marburg

andreas.diepold@mpi-marburg.mpg.de

www.diepoldlab.com

\section{AUTOREN}

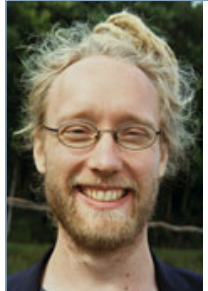

Stephan Wimmi

2009-2016 Biologiestudium an der TU Kaiserslautern und Universität Marburg. Masterarbeit am MaxPlanck-Institut für terrestrische Mikrobiologie, Marburg. 2017-2021 Promotion und seit 2021 Postdoc in der Arbeitsgruppe von Dr. A. Diepold.

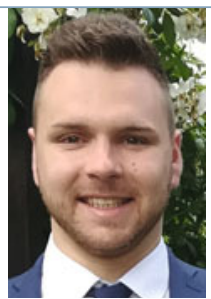

Florian Lindner

2013-2018 Molekularbiologiestudium an den Universiäten Göttingen und Marburg. 2017-2021 Masterarbeit und anschließende Promotion am Max-PlanckInstitut für terrestrische Mikrobiologie, Marburg, in der Arbeitsgruppe von Dr. A. Diepold.

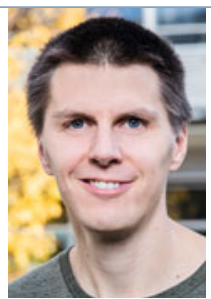

Andreas Diepold

Biochemiestudium an der Universität Tübingen, 2005 Diplomarbeit am Zenrum für Molekularbiologie der Pflanzen. 2005-2006 Novartis Institute for Biomedical Research. 2006-2011 Promotion und Postdoc am Biozentrum der Universität Basel. 20112017 Postdoc an der University of Oxford, UK. Seit 2017 Forschungsgruppenleiter am Max-Planck-Institut für terrestrische Mikrobiologie, Marburg.

\section{Hier steht eine Anzeige.}

